\title{
Vas Deferens Anastomotic Leakage
}

National Cancer Institute

\section{Source}

National Cancer Institute. Vas Deferens Anastomotic Leakage. NCI Thesaurus. Code C78704.

Leakage due to breakdown of a vas deferens anastomosis. 